\title{
Updown numbers and the initial monomials of the slope variety
}

\author{
Jeremy L. Martin* \\ Department of Mathematics \\ University of Kansas \\ Lawrence, KS 66047 USA \\ jmartin@math.ku.edu
}

\author{
Jennifer D. Wagner \\ Department of Mathematics and Statistics \\ Washburn University \\ Topeka, KS 66621, USA \\ jennifer.wagner1@washburn.edu
}

Submitted: May 28, 2009; Accepted: Jun 28, 2009; Published: Jul 9, 2009

Mathematics Subject Classifications: 05A15, 14N20

\begin{abstract}
Let $I_{n}$ be the ideal of all algebraic relations on the slopes of the $\left(\begin{array}{l}n \\ 2\end{array}\right)$ lines formed by placing $n$ points in a plane and connecting each pair of points with a line. Under each of two natural term orders, the ideal of $I_{n}$ is generated by monomials corresponding to permutations satisfying a certain pattern-avoidance condition. We show bijectively that these permutations are enumerated by the updown (or Euler) numbers, thereby obtaining a formula for the number of generators of the initial ideal of $I_{n}$ in each degree.
\end{abstract}

The symbol $\mathbb{N}$ will denote the set of positive integers. For integers $m \leq n$, we put $[n]=\{1,2, \ldots, n\}$ and $[m, n]=\{m, m+1, \ldots, n\}$. The set of all permutations of an integer set $P$ will be denoted $\mathfrak{S}_{P}$, and the $n^{\text {th }}$ symmetric group is $\mathfrak{S}_{n}\left(=\mathfrak{S}_{[n]}\right)$. We will write each permutation $w \in \mathfrak{S}_{P}$ as a word with $n=|P|$ digits, $w=w_{1} \ldots w_{n}$, where $\left\{w_{1}, \ldots, w_{n}\right\}=P$. If necessary for clarity, we will separate the digits with commas. Concatenation will also be denoted with commas; for instance, if $w=12$ and $w^{\prime}=34$, then $\left(w, w^{\prime}, 5\right)=12345$. The reversal $w^{*}$ of $w_{1} w_{2} \ldots w_{n-1} w_{n}$ is the word $w_{n} w_{n-1} \ldots w_{2} w_{1}$. A subword of a permutation $w \in \mathfrak{S}_{P}$ is a word $w[i, j]=w_{i} w_{i+1} \cdots w_{j}$, where $[i, j] \subseteq[n]$. The subword is proper if $w[i, j] \neq w$. We write $w \approx w^{\prime}$ if the digits of $w$ are in the same relative order as those of $w^{\prime}$; for instance, $58462 \approx 35241$.

Definition 1. Let $P \subset \mathbb{N}$ with $n=|P| \geq 2$. A permutation $w \in \mathfrak{S}_{P}$ is a $G$-word if it satisfies the two conditions

(G1) $w_{1}=\max (P)$ and $w_{n}=\max \left(P \backslash\left\{w_{1}\right\}\right)$; and

\footnotetext{
*Partially supported by an NSA Young Investigator's Grant
} 
(G2) If $n \geq 4$, then $w_{2}>w_{n-1}$.

It is an $R$-word if it satisfies the two conditions

(R1) $w_{1}=\max (P)$ and $w_{n}=\max \left(P \backslash\left\{w_{1}\right\}\right)$; and

(R2) If $n \geq 4$, then $w_{2}<w_{n-1}$.

A G-word (resp., an R-word) is primitive if for every proper subword $x$ of length $\geq 4$, neither $x$ nor $x^{*}$ is a G-word (resp., an R-word). The set of all primitive G-words (resp., on $P \subset \mathbb{N}$, or on $[n]$ ) is denoted $\mathcal{G}$ (resp., $\mathcal{G}_{P}$, or $\mathcal{G}_{n}$ ). The sets $\mathcal{R}, \mathcal{R}_{P}, \mathcal{R}_{n}$ are defined similarly.

For example, the word 53124 is a G-word, but not a primitive one, because it contains the reverse of the G-word 4213 as a subword. The primitive G-and R-words of lengths up to 6 are as follows:

$$
\begin{aligned}
\mathcal{G}_{2} & =\{21\} \\
\mathcal{G}_{3} & =\{312\} \\
\mathcal{G}_{4} & =\{4213\} \\
\mathcal{G}_{5} & =\{52314,53214\} \\
\mathcal{G}_{6} & =\{623415,624315,642315,634215,643215\} \\
\mathcal{R}_{2} & =\{21\} \\
\mathcal{R}_{3} & =\{312\} \\
\mathcal{R}_{4} & =\{4123\}, \\
\mathcal{R}_{5} & =\{51324,52134\} \\
\mathcal{R}_{6} & =\{614235,624135,623145,621435,631245\}
\end{aligned}
$$

Clearly, if $w \approx w^{\prime}$, then either both $w$ and $w^{\prime}$ are (primitive) G- (R-)words, or neither are; therefore, for all $P \subset \mathbb{N}$, the set $\mathcal{G}_{P}$ is determined by (and in bijection with) $\mathcal{G}_{|P|}$.

These permutations arose in [3] in the following way. Let $p_{1}=\left(x_{1}, y_{1}\right), \ldots, p_{n}=$ $\left(x_{n}, y_{n}\right)$ be points in $\mathbb{C}^{2}$ with distinct $x$-coordinates, let $\ell_{i j}$ be the unique line through $p_{i}$ and $p_{j}$, and let $m_{i j}=\left(y_{j}-y_{i}\right) /\left(x_{j}-x_{i}\right) \in \mathbb{C}$ be the slope of $\ell_{i j}$. Let $A=\mathbb{C}\left[m_{i j}\right]$, and let $I_{n} \subset A$ be the ideal of algebraic relations on the slopes $m_{i j}$ that hold for all choices of the points $p_{i}$. Order the variables of $A$ lexicographically by their subscripts: $m_{12}<m_{13}<$ $\cdots<m_{1 n}<m_{23}<\cdots$. Then [3, Theorem 4.3], with respect to graded lexicographic order on the monomials of $A$, the initial ideal of $I_{n}$ is generated by the squarefree monomials $m_{w_{1}, w_{2}} m_{w_{2} w_{3}} \cdots m_{w_{r-1} w_{r}}$, where $\left\{w_{1}, \ldots, w_{r}\right\} \subseteq[n], r \geq 4$, and $w=\left(w_{1}, w_{2}, \ldots, w_{r}\right)$ is a primitive G-word. Consequently, the number of degree- $d$ generators of the initial ideal of $I_{n}$ is

$$
\left(\begin{array}{c}
n \\
d+1
\end{array}\right)\left|\mathcal{G}_{d+1}\right|
$$

Similarly, under reverse lex order (rather than graded lex order) on $A$, the initial ideal of $I_{n}$ is generated by the squarefree monomials corresponding to primitive R-words. Our terms 
"G-word" and "R-word" denote the relationships to graded lexicographic and reverse lexicographic orders.

It was noted in [3, p. 134] that the first several values of the sequence $\left|\mathcal{G}_{3}\right|,\left|\mathcal{G}_{4}\right|, \ldots$ coincide with the updown numbers (or Euler numbers):

$$
1,1,2,5,16,61,272, \ldots
$$

This is sequence A000111 in the Online Encyclopedia of Integer Sequences [4. The updown numbers enumerate (among other things) the decreasing 012-trees [1, 2], which we now define.

Definition 2. A decreasing 012-tree is a rooted tree, with vertices labeled by distinct positive integers, such that (i) every vertex has either 0,1 , or 2 children; and (ii) $x<y$ whenever $x$ is a descendant of $y$. The set of all decreasing 012 -trees with vertex set $P$ will be denoted $\mathcal{D}_{P}$. We will represent rooted trees by the recursive notation $T=\left[v, T_{1}, \ldots, T_{n}\right]$, where the $T_{i}$ are the subtrees rooted at the children of $v$. Note that reordering the $T_{i}$ in this notation does not change the tree $T$. For instance, $[6,[5,[4],[2]],[3,[1]]]$ represents the decreasing 012-tree shown below.

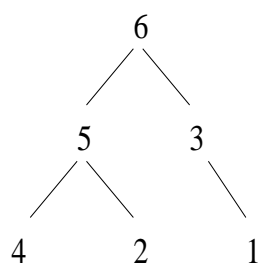

This notation differs slightly from [1] in that we do not require the largest or smallest vertex to belong to the last subtree listed. The reason for this is we would need one such convention in the context of G-words and a different one in the context of R-words, so we keep the notation more fluid here.

Our main result is that the updown numbers do indeed enumerate both primitive G-words and primitive R-words. Specifically:

Theorem 1. Let $n \geq 2$. Then:

1. The primitive $G$-words on $[n]$ are equinumerous with the decreasing 012-trees on vertex set $[n-2]$.

2. The primitive $R$-words on $[n]$ are equinumerous with the decreasing 012-trees on vertex set $[n-2]$.

Together with (2), Theorem 1 enumerates the generators of the graded-lex and reverselex initial ideals of $I_{n}$ degree by degree. For instance, $I_{6}$ is generated by $\left(\begin{array}{l}6 \\ 4\end{array}\right) \cdot 1=15$ cubic monomials, $\left(\begin{array}{l}6 \\ 5\end{array}\right) \cdot 2=12$ quartics, and $\left(\begin{array}{l}6 \\ 6\end{array}\right) \cdot 5=5$ quintics.

To prove Theorem 1, we construct explicit bijections between G-words and decreasing 012-trees (Theorem 7) and between R-words and decreasing 012-trees (Theorem 8). Our 
constructions are of the same ilk as Donaghey's bijection [2] between decreasing 012trees on $[n]$ and updown permutations, i.e., permutations $w=w_{1} w_{2} \cdots w_{n} \in \mathfrak{S}_{n}$ such that $w_{1}<w_{2}>w_{3}<\cdots$. In order to do so, we characterize primitive G-words by the following theorem. (Here and subsequently, the notation $(a, b) \in \mathfrak{S}_{P}$ serves as a convenient shorthand for the condition that $a$ and $b$ are (possibly empty) words on disjoint sets of letters whose union is $P$.)

Theorem 2. Let $n \geq 2$, and let $a, b$ be words such that $(a, b) \in \mathfrak{S}_{n-1}$. Then the word $(n+2, a, n, b, n+1) \in \mathfrak{S}_{n+2}$ is a primitive $G$-word if and only if $1 \in b$ and both $\left(n+1, a^{*}, n\right)$ and $(n+1, b, n)$ are primitive $G$-words.

In principle, there is a similar characterization for primitive R-words: if $(a, b) \in \mathfrak{S}_{n-1}$ and $\left(n+1, a^{*}, n\right)$ and $(n+1, b, n)$ are primitive $\mathrm{R}$-words, then either $(n+2, a, n, b, n+1)$ or $(n+2, b, n, a, n+1)$ is a primitive R-word; however, it is not so easy to tell which of these two is genuine and which is the impostor. (In the setting of G-words, the condition $1 \in b$ tells us which is which.)

Theorem 2 follows immediately from Lemmas 3] 6. which describe the recursive structure of primitive G- and R-words.

Lemma 3. Let $n \geq 3$ and let $w=\left(w_{1}, a, n-2, b, w_{n}\right) \in \mathfrak{S}_{n}$. Define words $w_{L}, w_{R}$ by

$$
w_{L}=\left(w_{1}, a^{*}, n-2\right), \quad w_{R}=\left(w_{n}, b, n-2\right) .
$$

Then:

1. If $w$ is a primitive G-word, then so are $w_{L}$ and $w_{R}$.

2. If $w$ is a primitive $R$-word, then so are $w_{L}$ and $w_{R}$.

Proof. We will show that if $w$ is a primitive G-word, then so is $w_{L}$; the other cases are all analogous. If $n=3$, then the conclusion is trivial. Otherwise, let $k$ be such that $w_{k}=n-2$. Then $2 \leq k \leq n-2$ by definition of a G-word. If $k=2$, then $w_{L}=w_{1} w_{2}$, while if $k=3$, then $w_{L}=w_{1} w_{3} w_{2}$; in both cases the conclusion follows by inspection. Now suppose that $k \geq 4$. Then the definition of $k$ implies that $w_{L}$ satisfies (G1), and if $w_{k-1}<w_{2}$ then $w[1, k]$ is a G-word, contradicting the assumption that $w$ is a primitive G-word. Therefore $w_{L}$ is a G-word. Moreover, $w_{L}[i, j] \approx w[k+1-j, k+1-i]^{*}$ for every $[i, j] \subsetneq[k]$. No such subword of $w$ is a G-word, so $w_{L}$ is a primitive G-word as desired.

Lemma 4. Let $n \geq 3$ and $x=\left(x_{1}, b, x_{n-1}\right) \in \mathfrak{S}_{n-1}$.

1. If $x$ is a primitive G-word, then so is

$$
w=(n, n-2, b, n-1)
$$

2. If $x$ is a primitive $R$-word, then so is

$$
w=\left(n, b^{*}, n-2, n-1\right) .
$$


Proof. Suppose that $x$ is a primitive G-word. By construction, $w$ is a G-word in $\mathfrak{S}_{n}$. Let $w[i, j]$ be any proper subword of $w$. Then:

- If $i \geq 3$, or if $i=2$ and $j<n$, then $w[i, j]=x[i-1, j-1]$ is not a G-word.

- If $i=2$ and $j=n$, then $w_{i}<w_{j}$ but $w_{i+1}=x_{2}>w_{j-1}=x_{n-2}$ (because $x$ is a G-word), so $w[i, j]$ is not a $\mathrm{G}$-word.

- If $i=1$, then $j<n$, but then $w_{i+1} \geq w_{j}$, so $w[i, j]$ is not a G-word.

Therefore $w$ is a primitive G-word. The proof of assertion (2) is similar.

Lemma 5. Let $n \geq 5$, and let $P, Q$ be subsets of $[n]$ such that

$$
p=|P| \geq 3, \quad q=|Q| \geq 3, \quad P \cup Q=[n], \quad \text { and } \quad P \cap Q=\{n-2\} .
$$

Let $x=\left(x_{1}, a, x_{p}\right) \in \mathfrak{S}_{P}$ and $y=\left(y_{1}, b, y_{q}\right) \in \mathfrak{S}_{Q}$ such that $x_{p}=n-2=y_{q}$ and $x_{p-1}>y_{q-1}$. Then:

1. If $x$ and $y$ are primitive $G$-words, then so is

$$
w=\left(n, a^{*}, n-2, b, n-1\right) .
$$

2. If $x$ and $y$ are primitive $R$-words, then so is

$$
w=\left(n, b^{*}, n-2, a, n-1\right) .
$$

Proof. Suppose that $x$ and $y$ are primitive G-words. By construction, $w$ is a G-word. We will show that no proper subword $w[i, j]$ of $w$ is a G-word. Indeed:

- If $i<p<j$, then $w[i, j]$ cannot satisfy (G1).

- If $i \geq p$, then either $[i, j]=[p, n]$, when $w_{i}=n-2<w_{j}=n-1$ and $w_{i+1}=$ $y_{2} \geq w_{j-1}=y_{q-1}$ (because $y$ is a G-word), or else $[i, j] \subsetneq[p, n]$, when $w[i, j] \approx$ $y[i-p+1, j-p+1]$. In either case, $w[i, j]$ is not a $\mathrm{G}$-word.

- Similarly, if $j \leq p$, then either $[i, j]=[1, p]$, when $w_{i}>w_{j}$ and $w_{i+1}=x_{p-1} \leq w_{j-1}=$ $x_{2}$ (because $x$ is a G-word), or else $[i, j] \subsetneq[1, p]$, when $w[i, j]^{*} \approx x[p-j+1, p-i+1]$. In either case, $w[i, j]$ is not a $\mathrm{G}$-word.

Therefore, $w$ is a primitive G-word. The proof of assertion (2) is similar.

The following and last lemma applies only to G-words and has no easy analogue for $\mathrm{R}$-words. As mentioned in the earlier footnote, this is why we characterize only primitive G-words and not primitive R-words in Theorem 2.

Lemma 6. Let $n \geq 2$ and let $w \in \mathcal{G}_{n}$. Then $w_{n-1}=1$. 
Proof. For $n \leq 4$, the result is easy to check due to the small number of G-words (see also (10). Otherwise, let $i$ be such that $w_{i}=1$. Note that $i \notin\{1,2, n\}$ by the definition of G-word. Suppose that $i \neq n-1$ as well. First, assume that $w_{i-1}<w_{i+1}$. Let $P=\left\{j \in[1, i-2] \mid w_{j}>w_{i+1}\right\}$. In particular $\{1\} \subseteq P \subseteq[1, i-2]$. Let $k=\max (P)$. Then

$$
\begin{aligned}
w_{k} & =\max \left\{w_{k}, w_{k+1}, \ldots, w_{i+1}\right\}, \\
w_{i+1} & =\max \left\{w_{k+1}, \ldots, w_{i+1}\right\}, \\
w_{k+1} & >w_{i}=1
\end{aligned}
$$

So $w[k, i+1]$ is a G-word. It is a proper subword of $w$ because $i+1 \leq n-1$, and its length is $i+2-k \geq i+2-(i-2)=4$. Therefore $w \notin \mathcal{G}_{n}$. If instead, $w_{i-1}>w_{i+1}$, then a similar argument shows that $w$ has a subword $w[i-1, k]$, where $i+2 \leq k \leq n$, whose reverse is a G-word.

For the rest of the paper, let $P$ be a finite subset of $\mathbb{N}$, let $n=|P|$, and let $m=\max (P)$. Define

$$
\begin{aligned}
\mathcal{G}_{P}^{\prime} & =\left\{w \in \mathfrak{S}_{P} \mid(m+2, w, m+1) \in \mathcal{G}\right\}, \\
\mathcal{R}_{P}^{\prime} & =\left\{w \in \mathfrak{S}_{P} \mid(m+2, w, m+1) \in \mathcal{R}\right\} .
\end{aligned}
$$

The elements of $\mathcal{G}_{P}^{\prime}$ (resp., $\mathcal{R}_{P}^{\prime}$ ) should be regarded as primitive G-words (resp., primitive R-words) on $P \cup\{m+1, m+2\}$, from which the first and last digits have been removed.

We now construct a bijection between $\mathcal{G}_{P}^{\prime}$ and the decreasing 012-trees $\mathcal{D}_{n}$ on vertex set $[n]$. If $P=\emptyset$, then both these sets trivially have cardinality 1 , so we assume henceforth that $P \neq \emptyset$. Since the cardinalities of $\mathcal{G}_{P}^{\prime}$ and $\mathcal{D}_{P}$ depend only on $|P|$, this theorem is equivalent to the statement that the primitive G-words on $[n]$ are equinumerous with the decreasing 012-trees on vertex set $[n-2]$, which is the first assertion of Theorem 11,

Let $w \in \mathcal{G}_{P}^{\prime}$ and $k$ be such that $w_{k}=m$. Note that if $n>1$, then $w_{n}<w_{1} \leq m$, so $k \neq n$. Define a decreasing 012-tree $\phi_{G}(w)$ recursively (using the notation of Definition 2) by

$$
\phi_{G}(w)= \begin{cases}{[m]} & \text { if } n=1 ; \\ {\left[m, \phi_{G}(w[2, n])\right]} & \text { if } n>1 \text { and } k=1 \\ {\left[m, \phi_{G}\left(w[1, k-1]^{*}\right), \phi_{G}(w[k+1, n])\right]} & \text { if } n>1 \text { and } 2 \leq k \leq n-1 .\end{cases}
$$

Now, given $T \in \mathcal{D}_{P}$, recursively define a word $\psi_{G}(T) \in \mathfrak{S}_{P}$ as follows.

- If $T$ consists of a single vertex $v$, then $\psi_{G}(T)=m$.

- If $T=\left[m, T^{\prime}\right]$, then $\psi_{G}(T)=\left(m, \psi_{G}\left(T^{\prime}\right)\right)$.

- If $T=\left[m, T^{\prime}, T^{\prime \prime}\right]$ with $\min (P) \in T^{\prime \prime}$, then $\psi_{G}(T)=\left(\psi_{G}\left(T^{\prime}\right)^{*}, m, \psi_{G}\left(T^{\prime \prime}\right)\right)$. 
For example, let $T$ be the decreasing 012-tree shown in Definition 2. Then

$$
\begin{aligned}
\psi_{G}(T) & =\psi_{G}([6,[5,[4],[2]],[3,[1]]]) \\
& =\left(\psi_{G}([5,[4],[2]])^{*}, 6, \psi_{G}([3,[1]])\right) \\
& =\left((452)^{*}, 6,31\right) \\
& =254631
\end{aligned}
$$

which is an element of $\mathcal{G}_{6}$ because, as one may verify, 82546317 is a primitive G-word. Meanwhile, $\phi_{G}(254631)=T$.

Theorem 7. The functions $\phi_{G}$ and $\psi_{G}$ are bijections $\mathcal{G}_{n}^{\prime} \rightarrow \mathcal{D}_{n}$ and $\mathcal{D}_{n} \rightarrow \mathcal{G}_{n}^{\prime}$ respectively.

Proof. First, we show by induction on $n=|P|$ that $\psi_{G}(T) \in \mathcal{G}_{P}^{\prime}$. This is clear if $n=1$; assume that it is true for all decreasing 012-trees on fewer than $n$ vertices.

If $T=\left[m, T^{\prime}\right]$, then $\psi_{G}(T)=\left(m, \psi_{G}\left(T^{\prime}\right)\right) \approx(n-2, a)$, where $a \in \mathfrak{S}_{n-3}$ and $a \approx \psi_{G}\left(T^{\prime}\right)$. By Lemma 圆 $(n, n-2, a, n-1) \approx\left(m+2, m, \psi_{G}\left(T^{\prime}\right), m+1\right)$ is a primitive G-word, and therefore $\psi_{G}(T) \in \mathcal{G}_{P}^{\prime}$.

If $T=\left[m, T^{\prime}, T^{\prime \prime}\right]$, then $\psi_{G}(T)=\left(\psi_{G}\left(T^{\prime}\right)^{*}, m, \psi_{G}\left(T^{\prime \prime}\right)\right) \approx\left(a^{*}, n-2, b\right)$, where $(a, b) \in$ $\mathfrak{S}_{n-3}$, with $a \approx \psi_{G}\left(T^{\prime}\right)$ and $b \approx \psi_{G}\left(T^{\prime \prime}\right)$. By Lemma 5 , therefore, $\left(n, a^{*}, n-2, b, n-1\right) \approx$ $\left(m+2, \psi_{G}\left(T^{\prime}\right)^{*}, m, \psi_{G}\left(T^{\prime \prime}\right), m+1\right)$ is a primitive G-word, and so $\psi_{G}(T) \in \mathcal{G}_{P}^{\prime}$.

Finally, showing that $\phi_{G}$ and $\psi_{G}$ are mutual inverses requires a technical but straightforward calculation, which we omit.

Next, we construct the analogous bijections for primitive R-words. Let $w \in \mathcal{R}_{P}^{\prime}$ with $k$ such that $w_{k}=m$. Note that if $n>1$, then $w_{1}<w_{n} \leq m$, so $k \neq 1$. Define a decreasing 012-tree $\phi_{R}(w)$ recursively by

$$
\phi_{R}(w)= \begin{cases}{[m]} & \text { if } n=1 ; \\ {\left[m, \phi_{R}\left(w[1, n-1]^{*}\right)\right]} & \text { if } n>1 \text { and } k=n ; \\ {\left[m, \phi_{R}\left(w[1, k-1]^{*}\right), \phi_{R}(w[k+1, n])\right]} & \text { if } n>1 \text { and } 2 \leq k \leq n-1 .\end{cases}
$$

Now, given $T \in \mathcal{D}_{P}$, we recursively define a word $\psi_{R}(T) \in \mathfrak{S}_{P}$ as follows.

- If $T$ consists of a single vertex $v$, then $\psi_{R}(T)=v$.

- If $T=\left[v, T^{\prime}\right]$, then $\psi_{R}(T)=\left(\psi_{R}\left(T^{\prime}\right)^{*}, v\right)$.

- If $T=\left[v, T^{\prime}, T^{\prime \prime}\right]$, and the last digit of $\psi_{R}\left(T^{\prime}\right)$ is less than the last digit of $\psi_{R}\left(T^{\prime \prime}\right)$, then $\psi_{R}(T)=\left(\psi_{R}\left(T^{\prime}\right)^{*}, v, \psi_{R}\left(T^{\prime \prime}\right)\right)$.

Again, if $T$ is the decreasing 012-tree shown in Definition 2 then

$$
\begin{aligned}
\psi_{R}(T) & =\psi_{R}([6,[3,[1]],[5,[4],[2]]]) \\
& =\left(\psi_{R}([3,[1]])^{*}, 6, \psi_{R}([5,[2],[4]])\right) \\
& =\left((13)^{*}, 6,254\right) \\
& =316254
\end{aligned}
$$

which is an element of $\mathcal{R}_{6}$ because, as one may verify, 83162547 is a primitive R-word. Meanwhile, $\phi_{R}(316254)=T$. 
Theorem 8. The functions $\phi_{R}$ and $\psi_{R}$ are bijections $\mathcal{R}_{n}^{\prime} \rightarrow \mathcal{D}_{n}$ and $\mathcal{D}_{n} \rightarrow \mathcal{R}_{n}^{\prime}$ respectively.

Proof. First, we show by induction on $n=|P|$ that $\psi_{R}(T) \in \mathcal{R}_{P}^{\prime}$. This is clear if $n=1$, so assume that it is true for all decreasing 012-trees on fewer than $n$ vertices.

If $T=\left[v, T^{\prime}\right]$, then $\psi_{R}(T)=\left(\psi_{R}\left(T^{\prime}\right), v\right) \approx\left(a^{*}, n-2\right)$, where $a \in \mathfrak{S}_{n-3}$ and $a \approx \psi_{R}\left(T^{\prime}\right)$. By Lemma [. $\left(n, a^{*}, n-2, n-1\right) \approx\left(v+2, \psi_{R}\left(T^{\prime}\right), v, v+1\right)$ is a primitive R-word, and therefore $\psi_{R}(T) \in \mathcal{R}_{P}^{\prime}$.

If $T=\left[v, T^{\prime}, T^{\prime \prime}\right]$, then $\psi_{R}(T)=\left(\psi_{R}\left(T^{\prime}\right)^{*}, v, \psi_{R}\left(T^{\prime \prime}\right)\right) \approx\left(b^{*}, n-2, a\right)$, where $(a, b) \in$ $\mathfrak{S}_{n-3}$ with $a \approx \psi_{R}\left(T^{\prime \prime}\right)$ and $b \approx \psi_{R}\left(T^{\prime}\right)$. By Lemma 1 therefore, $\left(n, b^{*}, n-2, a, n-1\right) \approx$ $\left(v+2, \psi_{R}\left(T^{\prime}\right)^{*}, v, \psi_{R}\left(T^{\prime \prime}\right), v+1\right)$ is a primitive $\mathrm{R}$-word, and so $\psi_{R}(T) \in \mathcal{R}_{P}^{\prime}$.

We have now constructed functions $\phi_{R}: \mathcal{R}_{n}^{\prime} \rightarrow \mathcal{D}_{n}, \psi_{R}: \mathcal{D}_{n} \rightarrow \mathcal{R}_{n}^{\prime}$. As in Theorem $\mathbf{7}$ we omit the straightforward proof that they are in fact mutual inverses.

\section{References}

[1] David Callan, A note on downup permutations and increasing 0-1-2 trees, http://www.stat.wisc.edu/ callan/notes/donaghey_bij/donaghey_bij.pdf, retrieved on May 28, 2009.

[2] Robert Donaghey, Alternating permutations and binary increasing trees, J. Combin. Theory Ser. A 18 (1975), 141-148.

[3] Jeremy L. Martin, The slopes determined by $n$ points in the plane, Duke Math. J. 131, no. 1 (2006), 119-165.

[4] N.J.A. Sloane, The On-Line Encyclopedia of Integer Sequences, 2008. Published electronically at www.research.att.com/ njas/sequences/. 